\title{
Biomechanical evaluation of fixation of comminuted olecranon fractures: one-third tubular versus locking compression plating
}

\author{
Geert A. Buijze $\cdot$ Leendert Blankevoort • \\ Gabriëlle J. M. Tuijthof • Inger N. Sierevelt • \\ Peter Kloen
}

Received: 16 February 2009/Published online: 13 October 2009

(C) The Author(s) 2009. This article is published with open access at Springerlink.com

\begin{abstract}
Introduction New concepts in plate fixation have led to an evolution in plate design for olecranon fractures. The purpose of this study was to compare the stiffness and strength of locking compression plate (LCP) fixation to one-third tubular plate fixation in a cadaveric comminuted olecranon fracture model with a standardised osteotomy.

Materials and methods Five matched pairs of cadaveric elbows were randomly assigned for fixation by either a contoured LCP combined with an intramedullary screw and unicortical locking screws or a one-third tubular plate combined with bicortical screws. Construct stiffness was measured by subjecting the specimens to cyclic loading while measuring gapping at the osteotomy site. Construct strength was measured by subjecting specimens to ramp load until failure.

Results There was no significant difference in fixation stiffness and strength between the two fixation methods. All failures consisted of failure of the bone and not of the hardware.

Conclusion Contoured LCP and intramedullary screw fixation can be used as an alternative treatment method for comminuted olecranon fractures as its stiffness and strength were not significantly different from a conventional plating technique.
\end{abstract}

G. A. Buijze $(\bowtie) \cdot$ L. Blankevoort · G. J. M. Tuijthof ·

I. N. Sierevelt

Orthopaedic Research Center Amsterdam,

Department of Orthopaedic Surgery, Academic Medical Center,

Meibergdreef 9, 1100 DD Amsterdam, The Netherlands

e-mail: G.A.Buijze@amc.uva.nl

P. Kloen

Department of Orthopaedic Surgery, Academic Medical Center, Meibergdreef 9, 1100 DD Amsterdam, The Netherlands
Keywords Locking compression plate - Comminuted · Olecranon fracture $\cdot$ Biomechanics $\cdot$ Cyclic loading

\section{Introduction}

Olecranon fractures are commonly seen in patients merely slipping or falling onto their arm as well as in cases of high energy trauma. In case of fracture comminution, stable and long-term reliable fixation is required. The reasons for these requirements are the necessity for immediate postoperative elbow motion for adequate rehabilitation and the risk of fatigue failure, which can be caused by extreme bending stresses [2]. Because of biomechanical advantages, plate fixation of these fractures is preferred over tension band wiring and therefore considered as the golden standard for treatment [5, 7-9]. Several authors have reported good clinical results by contouring a plate around the tip of the olecranon [1, 2, 9, 18, 21, 24]. Furthermore, placement of an intramedullary screw in a metaphyseal plate with bicortical screws has been reported to provide more support to the construct as it acts as an internal splint, analogous to an intramedullary nail [6].

As placement of an intramedullary screw may impede the placement of bicortical screws in the ulnar shaft, a locking compression plate (LCP) allowing for placement of unicortical screws can be used instead. If using unicortical locking screws, interference with a long intramedullary screw can be prevented. The biomechanical characteristics—such as stiffness and strength-of LCP fixation for (comminuted) olecranon fractures have not yet been evaluated.

The purpose of this biomechanical study is to compare LCP fixation to one-third tubular plate fixation in a simulated comminuted olecranon fracture using cyclic loading 
to measure the construct stiffness followed by destructive loading to determine the strength.

\section{Materials and methods}

Five matched pairs of fresh-frozen human cadaveric elbows, including the distal half of the humerus and the radius and ulna, were thawed at room temperature. There was one male specimen pair and four female specimen pairs with a mean age of 77.6 years (range 60-94 years). All soft tissue was removed except for the elbow joint capsule, medial and lateral collateral ligaments, annular ligament and triceps tendon. A small lateral capsulotomy was made to allow visual inspection of the joint for determining osteotomy location and reduction. At visual inspection of the specimens, no evidence of previous elbow injury in any of the tested elbow pairs was found.

To simulate a comminuted fracture, the specimens were secured in a custom-made jig (Fig. 1) using four parallel 2-mm K-wires. The ulna and radius were positioned along the long side of the jig, which had five vertical and two horizontal rows of 2.2-mm holes for insertion of $\mathrm{K}$-wires. The humerus was positioned in $90^{\circ}$ flexion along the lowest horizontal row of 2.2-mm holes. Three K-wires were placed perpendicular to the sagittal plane through the ulna using the holes in rows 1, 3 and 6 and one likewise through the distal humerus using a hole in row 7 (as illustrated in Fig. 1). The first oblique osteotomy was created with a fine-bladed saw from the posterior cortex (towards proximal) in the direction of the shallowest part of the trochlear notch using slot " $\mathrm{a}$ " and was completed using an osteotome. The second oblique osteotomy, towards distal, was created proximal to and joining the previous osteotomy at approximately half of the thickness of the bone using slot "b", creating a loose fragment. The simulated comminuted fracture was reduced and temporarily fixed by two parallel 2-mm K-wires from the tip of the olecranon into the shaft of the ulna.

One elbow from each pair $(n=5)$ was randomly assigned to a conventional plate fixation method as described by the AO-group [17]. An eight-hole one-third tubular plate (Synthes, Zeist, the Netherlands) was contoured around the tip of the olecranon and fixed by using five standard 3.5-mm bicortical screws (Fig. 2). The opposite elbow from each pair $(n=5)$ was fixed with a contoured eight-hole LCP (Synthes, Zeist, the Netherlands) using four $3.5-\mathrm{mm}$ self-tapping locking head screws and one $65-\mathrm{mm}$ long $3.5-\mathrm{mm}$ non-locking intramedullary screw (Fig. 3). In each pair of elbows, the screws were applied in similar configurations with one screw at the tip of the olecranon, one screw through and three screws distal to the fracture.

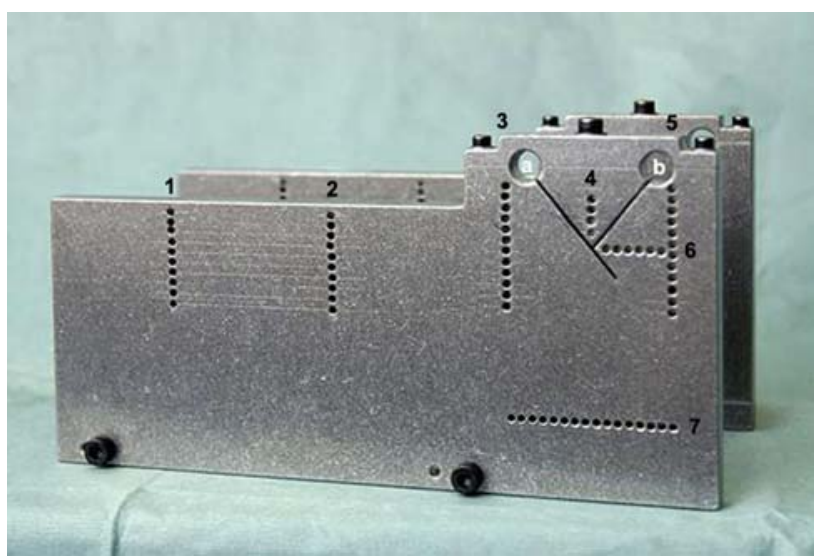

Fig. 1 The custom-made jig has small holes that were intended for insertion of 2-mm K-wires. To perform the osteotomy, the fine bladed osteotomy-saw was placed through the large holes and guided through the slits
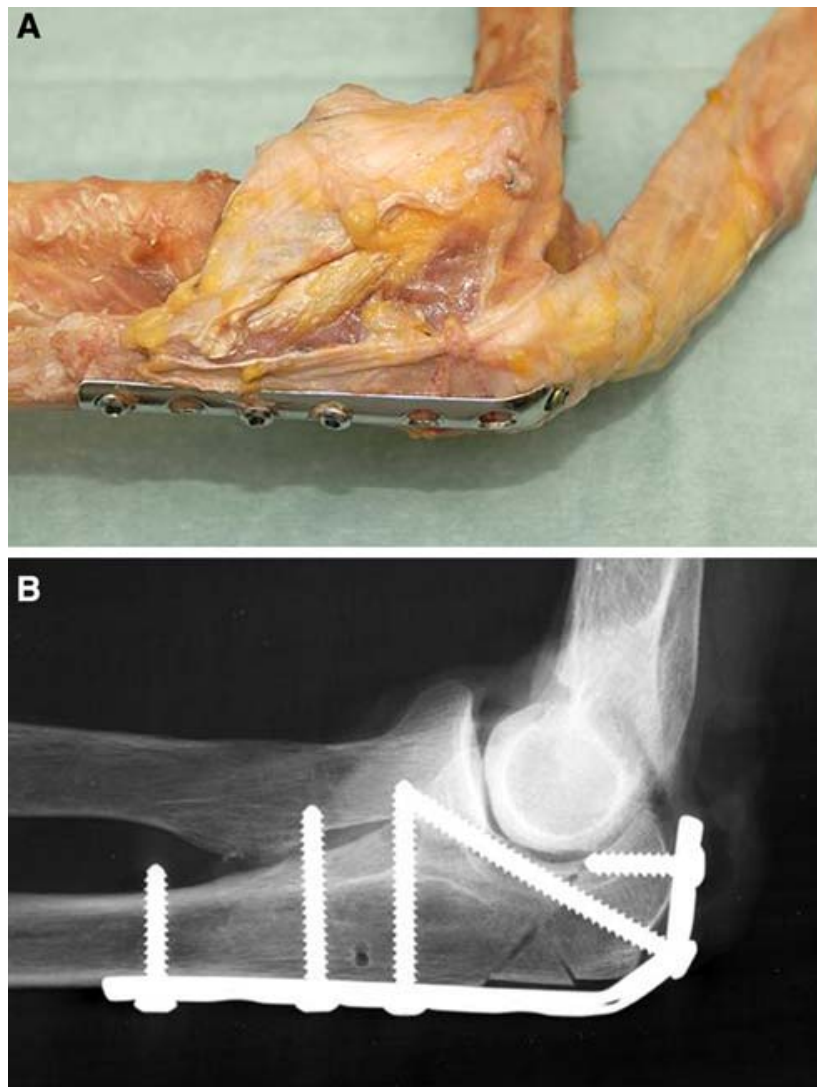

Fig. 2 a Medial view of specimen fixed by a one-third tubular plate showing the fixation with three bicortical screws (left), a coronoid lag screw (at the triceps attachment) and a short proximal screw (behind the triceps attachment). b One-third tubular plate fixation on lateral X-ray

The proximal humerus was potted into a polyvinyl chloride pipe with acrylic cement (Sulfix-6, Sulzer AG, Winterthur, Switzerland). The triceps tendon was secured by a custom-made stainless steel clamp. The pipe and 


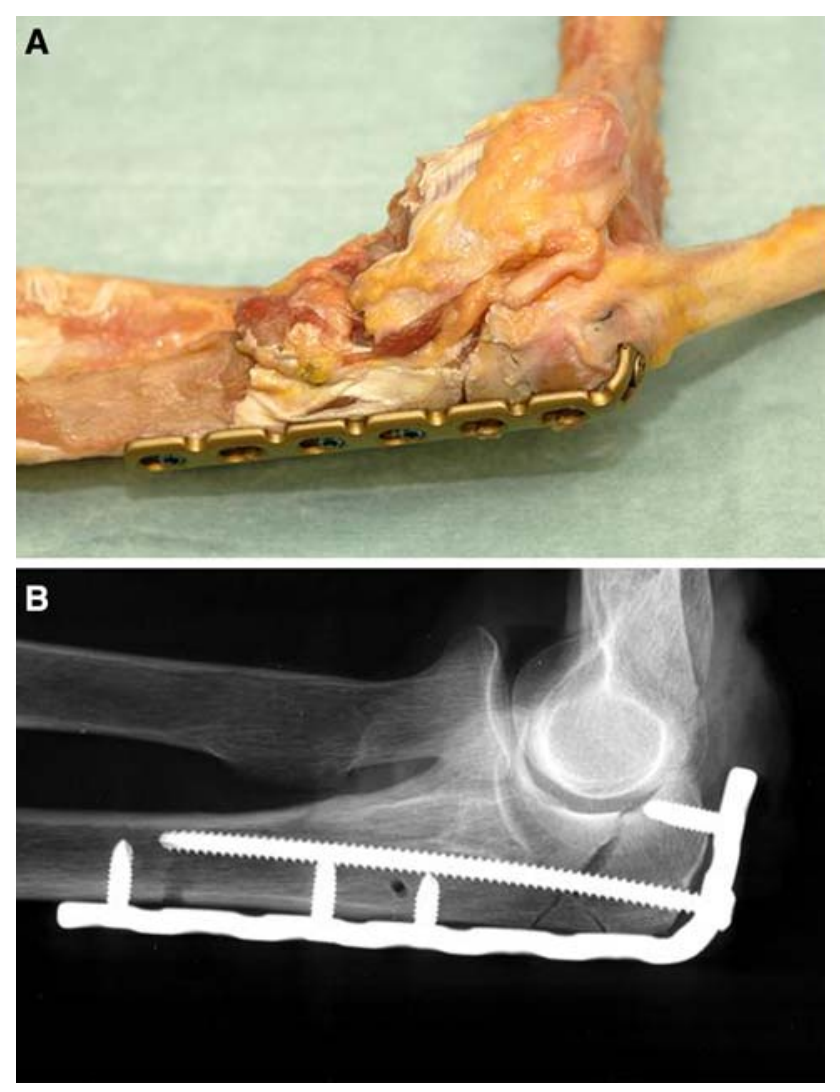

Fig. 3 a Medial view of a specimen fixed by a LCP showing the fixation with three unicortical screws distally, an intramedullary screw and a short proximal screw (behind the triceps attachment). Visible under the plate are the two olecranon osteotomy cuts that created a comminuted fracture. The triceps tendon is seen on the right. b LCP fixation on lateral X-ray

triceps clamps were mounted on a custom-made stepper motor actuated testing bench with a custom-made 3-component load cell attached to the actuator. The load cell had a range of 0 to $1,500 \mathrm{~N}$ and an accuracy of $\pm 0.4 \mathrm{~N}$. The elbow was flexed to $70^{\circ}$ (Fig. 4), similar to the setup of Fyfe et al. [5]. The testing bench was constructed by the Department of Medical Technical Development at our institution (Academic Medical Centre, Amsterdam, the Netherlands).

A differential variable reluctance transducer (DVRT; MicroStrain, Williston, VT, USA) with an accuracy of $\pm 0.01 \mathrm{~mm}$ was placed across the fracture at the posterior aspect of the olecranon, to measure fracture gapping during repetitive loading. The DVRT was secured in a custommade holder at approximately $11 \mathrm{~mm}$ above the ulnar cortex and mounted using two 1-mm diameter screws, one at each side of the fracture, lateral to the plate (Fig. 4).

By means of a prescribed vertical motion, the actuator of the testing bench, located at approximately $200 \mathrm{~mm}$ from the estimated joint axis, applied a saw-tooth shaped cantilever bending load to the distal part of the ulna. Before

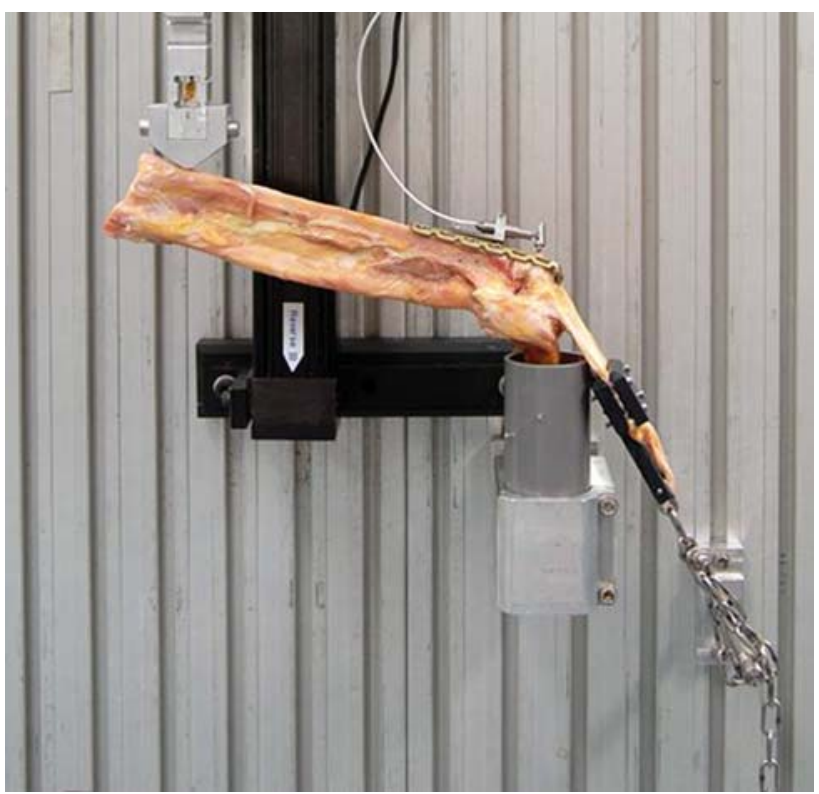

Fig. 4 The custom-made stepper motor actuated testing bench setup. The humerus is potted into a polyvinyl chloride pipe and the triceps tendon is secured with clamp and mounted on the bench. The DVRT is placed across the fracture at the posterior aspect of the olecranon. A bending moment is achieved at the osteotomy site by the actuator at the distal ulna

loading, the distal part of the actuator was positioned against the distal ulna without creating preload. No preload was applied to the triceps tendon other than the force required to counterbalance the effect of gravity on the ulna and radius. The force on the triceps tendon was not measured during testing as its moment was assumed to be equal to the moment caused by the force applied to the distal ulna. The actual distance between the actuator and the location of the fracture site was measured by using a ruler.

Two loading conditions were used to simulate both functional loading against mild resistance and a more aggressive push-up from a chair, similar to a previously published cyclic loading protocol [10]. During the first test run, ten cycles were performed in which the actuator forced a vertical distal ulnar motion of $60 \mathrm{~mm}$ at a velocity of $2 \mathrm{~mm} / \mathrm{s}$. The actuator returned to its starting position thereby completing a saw-tooth shaped displacement over time, which simulated the functional loading against mild resistance. During this test, the applied load at the distal ulna was measured by the load transducer with a sample frequency of $20 \mathrm{~Hz}$. At the second run, one load-to-failure test was performed in which the actuator forced a continuous uninterrupted vertical distal ulnar displacement at constant velocity. This caused an increasing load until the specimen failed catastrophically. Again, the load was measured during the complete run. This test simulated pushing up from a chair. The mode of failure was observed and noted in each specimen. 
The moment was determined for each specimen using the measurement of the distance from the mid-point of the fracture site to the location of the actuator. Stiffness was defined as the change in applied moment $(\mathrm{Nm})$ per unit of displacement at the fracture site $(\mathrm{mm})$. Stiffness was calculated as the slope of a linear fit through the loading phase of the last 5 cycles (Matlab v 7.5.0.342 (R2007b), the Mathworks, Natick, USA). One observer determined the end stiffness from the load-displacement data by analysing the linear part of the data starting from the highest load backward to the non-linear region, as indicated by a break point in the load-displacement curve (Fig. 5a). The slope of the linear fit through the data of the last 5 loading cycles was used to determine the stiffness per specimen (Fig. 5b). A coefficient of determination $\left(R^{2}\right)$ of more than 0.9 was considered valid. For determining the intra-observer reliability, the first observer repeated the procedure to calculate the stiffness. For the interobserver reliability a second independent observer repeated the procedure. The intraand interobserver reliability were estimated by the intraclass correlation coefficient (ICC) and the variability of the stiffness.

Load to failure criteria was defined as the moment required to create a gap of $2 \mathrm{~mm}$ at the fracture site or catastrophic failure, whichever came first. This gap displacement of $2 \mathrm{~mm}$ was chosen as a criterion for failure because intra-articular step-off larger than $2 \mathrm{~mm}$ has been associated with a higher incidence of posttraumatic arthrosis [15]. Catastrophic failure was defined as failure of the construct (e.g. hardware breakage or loosening) or failure of the bone (e.g. fracture of proximal fragments). Data of five pairs of specimens were collected and their results were compared. Since the sample size was small, a two-tailed Wilcoxon signed-ranks test was used for statistical analysis where $P<0.05$ was considered as a statistically significant difference.

\section{Results}

The stiffness of one LCP plated specimen could not be determined because of an error in registration of the displacement during cyclic loading. Therefore it was excluded from the analysis. The median stiffness for the one-third tubular plate plated specimens $(n=5)$ was $8.8 \mathrm{Nm} / \mathrm{mm}$ (range $6.2-33.8 \mathrm{Nm} / \mathrm{mm}$ ) (Table 1). The median stiffness for the LCP plated specimens $(n=4)$ was $13.3 \mathrm{Nm} / \mathrm{mm}$ (range $3.7-16.6 \mathrm{Nm} / \mathrm{mm}$ ) (Table 1). This difference was not statistically significant. The intraclass correlation coefficients for the intra- and interobserver reliability were both $0.99(P<0.0005)$. The absolute values for intra- and interobserver variability were 1.1 and $1.5 \mathrm{Nm} / \mathrm{mm}$, respectively. With failure defined as either $2 \mathrm{~mm}$ of gapping at the fracture site or catastrophic failure, the median load to failure criteria for the one-third tubular plate specimens $(n=5)$ was $11.3 \mathrm{Nm}$ (range 7.3-16.8 Nm). The median load to failure criteria for the LCP specimens $(n=5)$ was $10.8 \mathrm{Nm}$ (range $4.5-27.0 \mathrm{Nm}$ ). This difference was not statistically significant.

Even though the force on the distal ulna was converted to a cantilever bending moment at the anterior cortex of the fracture site, impaction of the anterior cortex was not seen in any specimen. Catastrophic failure did not occur in any specimen before the load that created a gap of $2 \mathrm{~mm}$ at the fracture site was reached. All catastrophic failures consisted of failure of the bone and not of the hardware. In one pair of specimens there was no catastrophic failure of the construct due to prior rupture of the triceps tendon, but the rupture occurred after the load with $2 \mathrm{~mm}$ gapping was reached. In the other specimens, the catastrophic failure mode was similar. Failure commenced at the midline of the olecranon between the two proximal screws, gradually splitting the olecranon into a lateral and medial fragment.

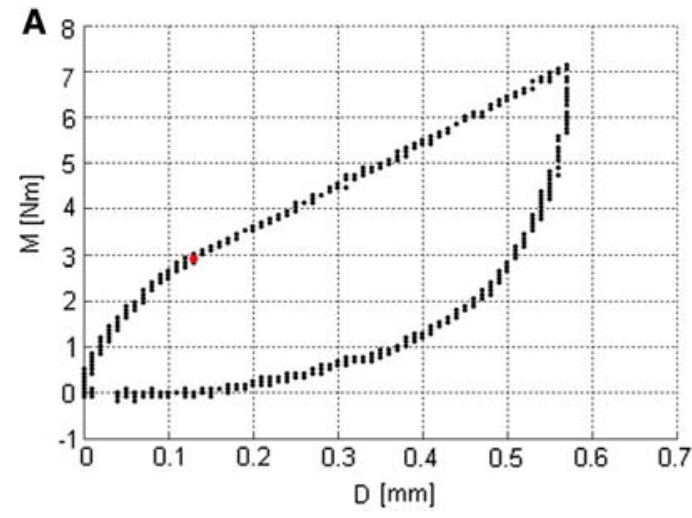

Fig. 5 a Sample graph showing the moment-displacement curve during a loading cycle (one-third tubular plate plated specimen, pair number 2). The red dot indicates the breakpoint of the starting force

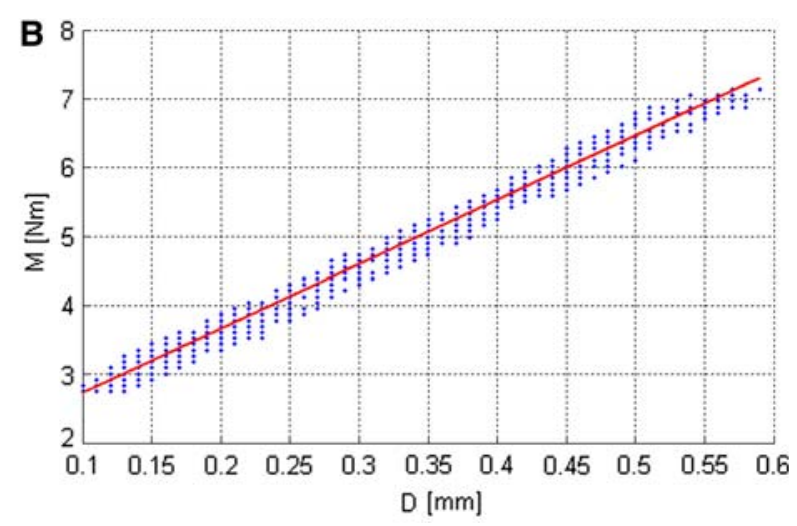

in the loading curve. b Sample graph showing a linear curve of best fit (red line) of the moment-displacement data of five cycles from this specimen 
Table 1 Load to failure criterion of $2 \mathrm{~mm}$ gapping, stiffness per specimen and coefficient of determination $\left(R^{2}\right)$ for the linear fit

\begin{tabular}{|c|c|c|c|c|c|c|c|c|c|c|}
\hline & \multicolumn{10}{|c|}{ Specimen pair } \\
\hline & \multicolumn{2}{|l|}{1} & \multicolumn{2}{|l|}{2} & \multicolumn{2}{|l|}{3} & \multicolumn{2}{|l|}{4} & \multicolumn{2}{|l|}{5} \\
\hline & TUB & LCP & TUB & LCP & TUB & LCP & TUB & LCP & TUB & LCP \\
\hline Load to failure criteria $(\mathrm{Nm})$ & 7.3 & 4.5 & 13.3 & 16.8 & 10.5 & 10.6 & 11.3 & 10.8 & 16.8 & 27.0 \\
\hline Stiffness $(\mathrm{Nm} / \mathrm{mm})$ & 26.0 & 3.7 & 8.8 & 16.6 & 6.2 & 11.3 & 8.1 & $-^{\mathrm{a}}$ & 33.8 & 15.3 \\
\hline$R^{2}$ & 0.95 & 0.99 & 0.97 & 0.99 & 0.97 & 0.98 & 0.98 & 0.41 & 0.98 & 0.95 \\
\hline
\end{tabular}

$T U B$ one-third tubular plate, $L C P$ locking compression plate

${ }^{\text {a }}$ No data, because of failed displacement measurement

\section{Discussion}

Stable fixation of olecranon fractures is essential as immediate postoperative rehabilitation has been shown to positively affect elbow range of motion and functional outcome [3, 20]. Moreover, hardware failure can occur due to extreme bending stresses on the plate construct when extending the elbow [21].

There have been previous reports on biomechanical strength of different plating techniques in comminuted olecranon fractures. To simulate a comminuted fracture, most studies were based on the creation of a gap (or segmental defect) at the osteotomy site, gap sizes varying up to $7 \mathrm{~mm}[6,11]$. It is unclear how well this correlates to a comminuted fracture without a large defect. In these simulations with segmental defects, there is no option for compression at fracture the site. Plates could only act as a buttress to prevent flexion bending at the fracture site. In other words, these studies merely tested the plate itself rather than the reconstruction. In this study, we aimed at creating a reproducible osteotomy model that would better simulate a comminuted fracture. However, this osteotomy model has not been previously evaluated.

In the current study, compression at the fracture site was achieved either by the coronoid lag screw in the one-third tubular plated specimens or by the intramedullary screw in the LCP specimens. The results reveal that there is no difference in stiffness or load to failure criterion of $2 \mathrm{~mm}$ gapping between locking compression plating and onethird tubular plating in comminuted olecranon fractures. However, several theoretical advantages have been reported on locking compression plating when compared to conventional plating in olecranon fractures, which have not been investigated in this study. First, it provides both angular and axial stability, which eliminates the need for exact plate contouring and thereby minimising the risk of primary loss of reduction. It acts as an "internal external fixator", which has shown to provide better rigidity because of its close proximity to the bone and fracture site [4]. Second, LCP fixations are not subject to the toggling of unlocked screws seen in conventional plates, which theoretically improves fixation in decreased bone mineral quality and comminution. However, this theoretical biomechanical advantage has not been proven in an osteoporotic bone model [22]. Furthermore, LCP preserves the periosteal blood supply, as no compression of the plate onto the bone is required, and generates better endosteal blood supply, as a part of the cortex is spared in unicortical screw fixation. Necrosis-induced bone loss as a consequence of decreased periosteal perfusion has been described as potential factor for implant loosening [16].

Higher construct stiffness does not imply superior outcome of osteosynthesis. Lill et al. [12] have reported on the early loosening and failure of the implant-bone interface caused by higher construct stiffness in cyclic loading of the proximal humerus. They suggest higher construct stiffness in LCP may lead to implant failure under physiological loading conditions. Although with lacking evidence in clinical trials, in our opinion the hypothetical increased risk of implant loosening due to excessive construct stiffness in vivo cannot be assumed.

There are limitations of this study with respect to the number of specimens and testing protocol. Testing results of matched pairs of specimens can be considered comparable, better than specimens between different donors. However, as some of the specimens were relatively old in our study and bone mineral density influences screw fixation, the lack of bone mineral density values presents a limitation of this study. There were large variations in absolute values for both stiffness and load to failure criterion of $2 \mathrm{~mm}$ gapping between the specimens. The largest range was found for the stiffness in the one-third tubular plating specimens, ranging between 6.2 and $33.8 \mathrm{Nm} / \mathrm{mm}$. The variability between cadavers is unpredictable, as has been reported in previous cadaveric studies $[11,13,14,19,23]$. Our findings are comparable to the findings of Gordon et al. [6] on construct stiffness for three plating methods in olecranon fractures, but should be interpreted with care, taking into account the methodological differences. Their mean stiffness values were $7.0 \mathrm{Nm} / \mathrm{mm}$ for dual ulna plating, $13 \mathrm{Nm} / \mathrm{mm}$ for posterior olecranon plating, and $17.6 \mathrm{Nm} / \mathrm{mm}$ for posterior olecranon plating with an intramedullary screw. These 
values are comparable to the median stiffness values found in our investigation, which were $8.8 \mathrm{Nm} / \mathrm{mm}$ for one-third tubular plating and $13.3 \mathrm{Nm} / \mathrm{mm}$ for LCP.

We performed our measurements in five elbows per group and this sample size was insufficient to find a significant difference. In order to achieve a statistical power of $80 \%$ with the present results and standard deviation, a sample size of 177 paired elbows would be required. Measurements of gapping were taken at location of the DVRT, placed at a distance of $11 \mathrm{~mm}$ above the posterior ulnar cortex. Displacement at the osteotomy site therefore is not equal to displacement at the transducer site. Therefore, failure defined in our study as displacement of $2 \mathrm{~mm}$ at the transducer site is not identical to the definition of a similar failure gap displacement by previous authors $[6,13]$. In addition, the "out-of-plane motions" at the osteotomy site were not taken into account as lateral and torsional motion of the ulna were assumed to be negligible in comparison to axial motion. Therefore, the DVRTmeasurement was representative only for the ulnar motion in the sagital plane at the osteotomy site. A better experimental design would be one in which all possible displacement directions, including torsional, compressive and lateral motions could be detected.

Taking account of the limitations of this study, no difference was found in fixation stiffness and strength between LCP fixation and one-third tubular plate fixation in a simulated comminuted fracture model of the olecranon. Contoured LCP and intramedullary screw fixation can be used as an alternative treatment method for comminuted olecranon fractures as its stiffness and strength were not significantly different from a conventional plating technique.

Acknowledgments The authors appreciate the support of Stichting Wetenschappelijk Onderzoek Orthopaedische Chirurgie for their financial contribution to the study, and the support of Synthes Holland in providing the internal fixation devices used for this study.

Open Access This article is distributed under the terms of the Creative Commons Attribution Noncommercial License which permits any noncommercial use, distribution, and reproduction in any medium, provided the original author(s) and source are credited.

\section{References}

1. Anderson ML, Larson AN, Merten SM, Steinmann SP (2007) Congruent elbow plate fixation of olecranon fractures. J Orthop Trauma 21:386-393

2. Bailey CS, MacDermid J, Patterson SD, King GJ (2001) Outcome of plate fixation of olecranon fractures. J Orthop Trauma 15:542548

3. Cabanela M, Morrey B (1993) Fractures of the proximal ulna and olecranon. In: Morrey BF (ed) The elbow and its disorders. WB Saunders, Philadelphia, pp 381-399
4. Egol KA, Kubiak EN, Fulkerson E, Kummer FJ, Koval KJ (2004) Biomechanics of locked plates and screws. J Orthop Trauma 18:488-493

5. Fyfe IS, Mossad MM, Holdsworth BJ (1985) Methods of fixation of olecranon fractures. An experimental mechanical study. J Bone Joint Surg Br 67:367-372

6. Gordon MJ, Budoff JE, Yeh ML, Luo ZP, Noble PC (2006) Comminuted olecranon fractures: a comparison of plating methods. J Shoulder Elbow Surg 15:94-99

7. Hak DJ, Golladay GJ (2000) Olecranon fractures: treatment options. J Am Acad Orthop Surg 8:266-275

8. Horner SR, Sadasivan KK, Lipka JM, Saha S (1989) Analysis of mechanical factors affecting fixation of olecranon fractures. Orthopedics 12:1469-1472

9. Hume MC, Wiss DA (1992) Olecranon fractures. A clinical and radiographic comparison of tension band wiring and plate fixation. Clin Orthop Relat Res 285:229-235

10. Hutchinson DT, Horwitz DS, Ha G, Thomas CW, Bachus KN (2003) Cyclic loading of olecranon fracture fixation constructs. J Bone Joint Surg Am 85-A:831-837

11. King GJ, Lammens PN, Milne AD, Roth JH, Johnson JA (1996) Plate fixation of comminuted olecranon fractures: an in vitro biomechanical study. J Shoulder Elbow Surg 5:437-441

12. Lill H, Hepp P, Korner J, Kassi JP, Verheyden AP, Josten C et al (2003) Proximal humeral fractures: how stiff should an implant be? A comparative mechanical study with new implants in human specimens. Arch Orthop Trauma Surg 123:74-81

13. Molloy S, Jasper LE, Elliott DS, Brumback RJ, Belkoff SM (2004) Biomechanical evaluation of intramedullary nail versus tension band fixation for transverse olecranon fractures. J Orthop Trauma 18:170-174

14. Mullett JH, Shannon F, Noel J, Lawlor G, Lee TC, O'Rourke SK (2000) K-wire position in tension band wiring of the olecranona comparison of two techniques. Injury 31:427-431

15. Murphy DF, Greene WB, Dameron TB Jr (1987) Displaced olecranon fractures in adults. Clinical evaluation. Clin Orthop Relat Res 224:215-223

16. Perren S (1995) Point contact fixator: Part I. Scientific background, design and application. Injury 22:1-10

17. Quintero J (2000) Olecranon/radial head/complex elbow injures. In: Rüedi TP, Murphy WM (eds) AO Principles of fracture management. Thieme, Stuttgart, pp 323-339

18. Ring D, Jupiter JB, Sanders RW, Mast J, Simpson NS (1997) Transolecranon fracture-dislocation of the elbow. J Orthop Trauma 11:545-550

19. Sanders R, Haidukewych GJ, Milne T, Dennis J, Latta LL (2002) Minimal versus maximal plate fixation techniques of the ulna: the biomechanical effect of number of screws and plate length. J Orthop Trauma 16:166-171

20. Scharplatz D, Allgöwer M (1975) Fracture-dislocations of the elbow. Injury 7:143-159

21. Simpson NS, Goodman LA, Jupiter JB (1996) Contoured LCDC plating of the proximal ulna. Injury; 27:411-417

22. Snow M, Thompson G, Turner PG (2008) A mechanical comparison of the locking compression plate (LCP) and the low contact-dynamic compression plate (DCP) in an osteoporotic bone model. J Orthop Trauma 22(2):121-125

23. Tejwani NC, Garnham IR, Wolinsky PR, Kummer FJ, Koval KJ (2002-2003) Posterior olecranon plating: biomechanical and clinical evaluation of a new operative technique. Bull Hosp Jt Dis 61:27-31

24. Waddell G, Howat TW (1973) A technique of plating severe olecranon fractures. Injury 5:135-140 